\title{
WHIM syndrome
}

INSERM

\section{Source}

INSERM. (1999). Orphanet: an online rare disease and orphan drug data base. WHIM syndrome. ORPHA:51636

WHIM (warts, hypogammaglobulinemia, infections, and myelokathexis) syndrome is a congenital autosomal dominant immune deficiency characterized by abnormal retention of mature neutrophils in the bone marrow (myelokathexis) and occasional hypog ammag lobulinemia, associated with an increased risk for bacterial infections and a susceptibility to human papillomavirus (HPV) induced lesions (cutaneous warts, genital dysplasia and invasive mucosal carcinoma). 